\title{
Sistema de Laboratorios a Distancia [SLD]: laboratorio para la enseñanza del control automático a distancia
}

Aldo R. Sartorius Castellanos

Luis Hern·ndez Santana Rafael Aracil Santonja Ernesto Rubio Rodrlyuez Iv.n Santana Ching

\section{Remote Laboratory Systems [RIS]: Learning laboratory in the automatic controlat distance}


Resumen: En este trabajo es presentado el Sistema de Laboratorios a Distancia (SLD), el cual es un laboratorio a distancia enfocado al estudio del control autom tico desarrollado en la Universidad Central ìMarta Abreuî de las Villas. El principal objetivo de este laboratorio es permitir a los usuarios aprender a ajustar controladores predefinidos y a diseÒar sus propios controladores para posteriormente probarlos sobre un conjunto de dispositivos fìsicos a travÈs de Internet. El SLD utiliza herramientas de diseÒo de sistemas de control asistido por computadora como Matlab-Simulink para la creaciÛn de nuevos controladores, lo que facilita a los usuarios el uso del sistema. EI SLD permite una $r \cdot p i d a y ~ f \cdot c i l$ integraciÛn de nuevos procesos para experimentos de control y en la actualidad est. $n$ disponibles tres dispositivos: un motor de corriente directa y un robot manipulador en Cuba asì como un cilindro electro neum·tico en EspaÒa.

Palabras clave: EducaciÛn a distancia. Laboratorio real. Laboratorio virtual. Sistemas de control.

Abstract: This work aims at presenting the Remote Laboratories System (RLS), a virtual and remote laboratory, in connection with the automatic control laboratory developed at the Universidad Central "Marta Abreu" de las Villas. The main objective of this laboratory is to allow users to learn how to regulate predefined controllers and later to design their own controllers in order to test them on a set of physical devices through the Internet. The RLS uses computer assisted design control systems tools - like the Matlab-Simulink - in new controllers creation, makes it easier to users when utilizing the system. The RLS allows a fast and easy integration of new processes with control experiments and, at present, three devices are available: a $R C$ engine and a manipulator robot in Cuba, as well as an electricpneumatic cylinder in Spain.

Keywords: Distance Education, Remote Laboratory, Virtual Laboratory, Control Systems

CASTELLANOS,AldoR.Sartoriusetal.SistemadeLaboratorios aDistancia(SLD):LaboratorioparalaenseÒanzadelcontrol autom tico a distancia. Informática na Educação: teoria \& prática, Porto Alegre, v.7, n. 1, p.11-24, jan./jun. 2004. 


\section{Introducción}

En los 'Itimos aÒos se ha presentado un cambio dram tico en la educaciÛn debido principalmente a Internet y a las tecnologlas Web. La enseÒanza basada en Web, los cursosadistancia, los libroselectrûnicosylasplataformasinteractivas deeducaciÛn adistancia juegan un papel cadavez m.simportanteenel procesodeaprendizaje.EnAlhalabietal.(1998), elautoranalizaelestadodelartedelaeducaciÛn a distancia. Un estudio sobre los sistemas educacionales basados en Webes proporcionado en Ausserhofer (1999), donde se realiza una descripciÛn detallada de los problemas actuales concernientes al diseÒo de sistemas educacionales basadosen Webysusposibles soluciones. EnShor(2000), elautorproporciona algunas recomendaciones en cuanto a las caracterl̀sticasquedebentenerloslaboratorios a distancia, adem.s de analizar los beneficios econÛmicosy decooperaciÛnque representa su uso. El rea de control autom tico es una de las -reas tÈcnicas que m.s ha explotado las nuevas tecnologìs para desarrollar herramientasque faciliten el aprendizaje a distancia(CASINI,etal.,2003). Unprofundoan-lisis sobrelaenseÒanzadelcontrolautom·ticousando las nuevas tecnologlas Web es reportado enSebasti-n (2002). En Poindexteretal.(1999), Poindexter y Heck presentan unagula parautilizar los sistemas de educaciûn a distancia basados en Weben el - rea del control autom-tico. En este artìculo los autores prestan especial atenciÛn a los laboratorios a distancia que son accesibles a travĖs del Web. Los laboratorios a distancia basados en Web se pueden clasificar en dos grandes clases: virtuales y reales. Loslaboratoriosa distancia virtualesse basan en la simulaciÛn de sistemas fìsicos de formaremota. EnellosatravÈs delaanimaciûn por computadora y el uso de software especializadose pueden representarestossistemas fìsicos de formagraficay analìtica. En laactualidad es uno de los mÈtodos mas utilizados en los programas de enseÒanza a distancia basados en Web(ALHALABI, etal., 2000). En Schmid (1998), se presenta un laboratorio virtualque haceusodedos plug-in, uno paravisualizar los datos en el navegador del clientey otro paracomunicarse con Matlabenel servidor remoto. En Chaudhuri et al. (2003), se presenta un laboratorio virtual que hace uso de las potentes capacidades de LabVIEW para implementarunlaboratoriovirtual de controlde procesos.

Los laboratorios a distancia reales en cambio, permiten a los usuarios interactuar con dispositivos reales a travÈs de Internet. Normalmente los usuarios a travÈs de una interfaz Web pueden cambiar algunos par-metros decontrol, realizarlosexperimentos, ver los resultadosy descargar los datos delexperimento. Este tipo de experimentos son presentados porejemplo en (KO, etal., 2001), dondeunlaboratorioadistanciareal paracontrol denivelentanquesesmostrado;enDomìnguez et al. (2001), es descrito un laboratorio remoto para el control de procesos; y en Hahn et al., (2000) est $\cdot n$ disponibles varios experimentos con sistemas mec.nicos.

LacomplejidadeneldiseÒodel hardware y principalmente del software se incrementa $\mathrm{dr}$-sticamente en los sistemas que permiten a los usuarios diseÒar controladores de forma remota. Un ejemplo de esto es el caso del sistema presentado en Chiculita et al. (2002), en donde los usuarios pueden controlar de forma remotael nivelde untanque, modificaralgunos par-metrosy transmitir el programa de control, el cual cambiara la din.mica en lazo cerrado del sistema. En este laboratorio los usuarios pueden desarrollarsus propios controladores utilizando el lenguaje Phyton. Este cÛdigo es 
enviado al servidor del laboratorio remoto en dondeescompiladoyejecutadoparacontrolar el proceso. Lainterfaz de usuario de estesistemafue desarrolladacomo una aplicaciÛngr.fica que utiliza el protocolo TCP/IP para comunicarseconelservidor. Unenfoquediferentees presentado por Timothy Changy Douglas Hung en Chang, et al. (2000), en donde los usuarios puedenimplementarsuspropioscontroladores deformaremota, utilizando unconjunto defunciones desarrolladas en lenguaje $C$. EstecÛdigo es compilado por el servidor del laboratorio remotoycargadoen unatarjetaprovistaconun procesador digital de seÒales que se encarga decontrolarunmotorconunpÈnduloinvertido.

En general los laboratorios a distancia utilizan software ampliamente conocido como LabVIEW(TREVELYAN,2002)y Matlab-Simulink (DIXON, etal., 2001, JUNGE, etal., 2000), pero tambiÈn algunos sistemas utilizan software especifico desarrollado para este fin, tal es el caso del sistema propuesto por Frangu y Chiculita en Frangu et al. (2002), donde un proceso de controldenivelespresentado. Este artìculodescribeeldiseÒoy construcciÛndeun laboratorio a distancia virtualy realenfocadoal estudio del control autom tico que ha sido desarrollado en la Universidad Central ìMarta Abreuî de las Villas. EI SLD permite a los usuarios cambiar las referencias, modificarlos par-metros de control, variar el tiempo de muestreoy diseÒarsus propios controladores de unaforma sencilla, utilizando herramientas ampliamente conocidas en el - rea del control autom·tico como son Matlaby Simulink. Laprincipal caracterl̀stica deISLDesquelosusuarios del sistema pueden crear controladores complejos, que no est $\cdot n$ restringidos a los bloques provistos por Simulink, ya que se puedenimplementarcontroladoresqueutilicen sus propios bloques de Simulink, creados a travĖs deS-Functions definidas por el usuario en lenguaje C. Los autores piensan que un punto muy importante es que el sistema es accesible a todos los usuarios de una forma sencilla, utilizandounacomputadoraconectada a Internet y cualquier navegador Web, sin necesidad de descargar o instalar ning $\mathrm{n}$ software adicional paraello.

Este artìculo es organizado de la siguiente forma: La secciÛn 2 describe las caracterl̀sticascomunesyespecificas delSLDasì como algunas notas sobre su funcionamiento general. La secciÛn 3 describe el uso del SLD desde un punto de vista pr.ctico. La secciÛn 4 explica la creaciÛn de controladores por parte de los usuarios. En la secciûn 5 se describe la administraciÛndelsistema. EnlasecciÛn6son comentadas algunas experiencias del uso del SLD mientras que las conclusiones son mostradas en la secciÛn 7 .

\section{Características del sistema}

EISLD es un laboratorio a distancia virtual y real, cuyo principal objetivo es permitir a los usuarios aprender a ajustar controladores predefinidos y a diseÒar sus propios controladores para probarlos en dispositivos reales que sonaccesiblesatravÈs de Internet.

\subsection{Características comunes de los laboratorios a distancia}

EI SLD posee algunas caracterìsticas comunesconlagranmayorladeloslaboratorios a distancia implementados actualmente (BAUCHSPIESS, etal.,2003), (GARCÕA, etal., 2003), tales como:

Disponibilidad: Los sistemas de enseÒanza basados en Web deben de poder estar disponibles las 24 horas del dìa. Esto implica que el sistema debe de tener medidas deautoprotecciÛn paragarantizaresteaspecto. Todos los experimentos deben de ser equipa- 
dos con dispositivos hardware y software que prevengan daÒos al equipo o al personal presenteenellaboratorio.

Accesibilidad: Debido a que el SLD esta montado sobre una plataforma Web, permite a los usuarios acceder al sistema desde cualquier parte del mundo. Para ello solo es necesaria una computadora con conexiÛn a Internet y un navegador Web, tales como el Internet Explorero Netscape Navigator.

Facilidad de uso: Para utilizar el SLD los usuarios solo deben tener los conocimientos b-sicos de los sistemas de control, tales como el modelado de dispositivos fìsicos y el diseÒo de controladores. De esta forma el usuario se centra en aprender estos temas y evita todos los problemas asociados a la implementaciÛn y operaciÛn de los equipos usados en las pr-cticas.

\subsection{Características específicas del SLD}

Adem.s delas caracterl̀sticas descritas anteriormente, las cuales son comunes a la mayorla deloslaboratorios virtualesyremotos, el SLD posee caracterìsticas adicionales que soncomentadasacontinuaciûn:

Interfaz rápida y fácil: Una parte muyimportante en el desarrollo de un sistema de enseÒanza basado en Web es la interfaz de usuario. La principal funciÛn de esta parte del sistemaesconformarel pedido de las pr.cticas ymandarlohaciaelservidor Web. Lainterfazde usuario delSLD estabasada en p.ginas htmly ASP; esto permite que los usuarios puedan acceder al sistema de una forma r.pida y sin necesidad de descargar o instalar ning $n$ softwareadicional. Elsistemacuentatambièn con p.ginas de ayuda que proporcionan informaciÛntÈcnica a los usuarios, tal como el modelado matem tico de los dispositivos usa- dosenlaspr-cticas, datos delfabricanteyajuste de reguladores entre otros tÛpicos de interÈs paralas pr.cticas.

Administración de múltiples pedidos en forma paralela: EI SLD es capaz de atender $\mathrm{m}$ ' Itiples pedidos de pr.cticas de forma paralela, administrando centralizadamente, dispositivos similaresque se encuentrengeogr-ficamenteseparadosperounidosporredes de -reaextensa. Esto permite unamayordisponibilidad de los equipos y un servicio $\mathrm{m} \cdot \mathrm{s} r$. pido y eficiente para los usuarios, logr $\cdot$ ndose con estoreducir lostiempos deesperaparaqueun usuario realice una determinadapr $\cdot$ ctica.

Desarrollo de controladores de forma remota utilizando Matlab y Simulink: Una de las caracterl̀sticas $\mathrm{m} \cdot \mathrm{s}$ importantes del SLD, es que posibilita a los usuarios diseÒar sus propios controladores utilizando el ambiente Matlab-Simulink. Estos programas son un est.ndar en el ·rea del control autom tico, por lo que los usuarios no necesitan perder tiempo aprendiendo nuevos lenguajes de programaciÛnparaimplementaruncontrolador nuevo, solonecesitan unconocimiento b-sico delambiente de Matlaby Simulink. A travÈs de lainterfazgraficade Simulink, unagrancantidad de bloques pueden ser seleccionados y conectados $f \cdot c i l m e n t e$, lo que permite a los usuarios crear controladores analÛgicos, digitales o hìbridos de una forma muy $r$-pida. Adicionalmente, el SLD no restringe a los usuarios a usar solo los bloques provistos por Simulink, sino que permite de forma opcional, la creaciÛn de controladores complejos que incluyan S-Functions definidas por el usuario. Estas S-Functions deben ser realizadas utilizando el lenguaje de programaciÛn C, el cual es un lenguaje muy potente y ampliamente conocido por la mayorla de los estudiantes de ingenierla enlaactualidad. 
Tipo de controlador:Todos los experimentos en el SLD pueden ser controlados de dos formas distintas: con controlador predefinido o con controlador definido por el usuario. En el primer caso los usuarios deben asignar los valores a las distintas ganancias del controlador, por ejemplo el usuario puede escoger un controlador PID y cambiar los par-metros de las ganancias proporcional, integral y derivativa para ver la respuesta del sistema. En el segundo caso los usuarios pueden diseÒar su propio controlador (como se ha explicado en el punto anterior) y enviarlo al SLD donde ser implementado y usado para controlar el experimento.

Cambio del periodo de muestreo: EISLD permite a los usuarios variar el periodo de muestreo de los experimentos. Esto permite examinar como afecta este par-metro en el diseÒo de controladores digitales y en general en el desempeÒo de cualquier experimento.

Cambio de referencias: Elsistemapermite variar las referencias de los experimentos para poder evaluar el comportamiento de un determinado controlador ante distintas seÒales de entrada.

\subsection{Funcionamiento general DEL SLD}

EISLD se divide en tres partes:

1.-Interfazdelusuario

2.-AdministraciÛn delospedidos de las pr·cticas.

3. - Procesamiento de las pr-cticas.

LaFigura 1 muestralainteracciÛndeestas tres partes. Los usuarios interact an conel sistema a travÈs de Internet. El sitio Web del sistema estatotalmente desarrollado utilizando el lenguaje html y ASP. Al acceder al sitio

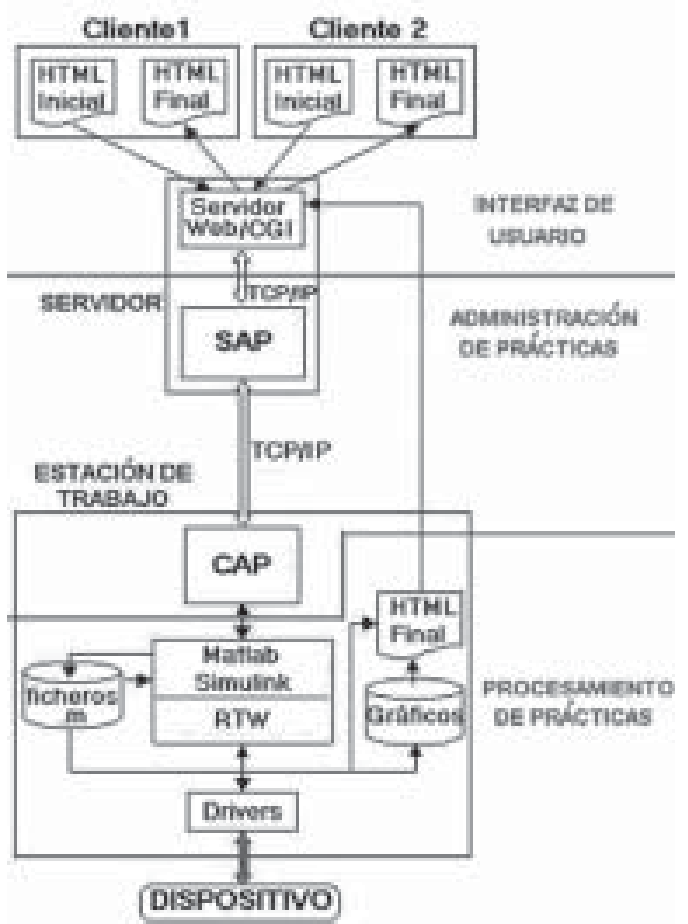

Figura 1: Arquitectura utilizada a nivel de software y de hardware en el Sistema de Laboratorios a Distancia (SLD)

Web, el usuario debe autentificarsey posteriormente elegir la pr-ctica que se desea realizar. Alli elusuario debellenarcorrectamentetodos los datos en el formulario asociado a la pr ctica y finalmente elegir entre ejecutarla de manera simulada o real. El CGI que se encuentra en el servidor Webrecibe los datosy los envla hacia el Servidor de Administración de Prácticas(SAP) comounnuevopedido. EISAPverificacualestaciÛn de trabajo puede realizar la pr·ctica pedida, yunavezquelaencuentra, extraeel pedido delalistay se lo envìa al Cliente de Administración de Prácticas(CAP) instaladoenlaestaciÛn de trabajo. Cuando el pedido llega al CAP se procesan los datos y selleva a cabolapr-ctica utilizando Matlab-Simulink. Una vez que la pr.ctica hasido procesadase trasmiteel resultado en sentido inverso al que trajo el pedido, 
para que al final Ilegue hasta el usuario la respuesta de la pr·ctica. La respuesta es una $\mathrm{p} \cdot \mathrm{gina}$ Web que muestra los resultados del procesamiento.

Elservidor WebyelSAP seencuentran instalados en una computadora que utiliza Windows 2000 como sistema operativo. Las estaciones de trabajo necesitan tener instalado el CAP asì como Matlab y Simulink para el procesamiento de las pr.cticas. Las estaciones de trabajo utilizan Windows 98como sistemaoperativo.

El sistema cuenta actualmente con tres procesos para realizar experimentos: un motor de corriente directa, un robot manipulador Asea IRB-6y un cilindro electroneum-tico. Elmotordecorrientedirectaesun procesomuy simplede una sola entraday una sola salida, el cual nospermite realizarexperimentos deidentificaciÛn, controldeposiciÛnydevelocidadsin disturbios decarga. Elcilindroelectroneum tico es tambièn un proceso de una entrada y una salida pero que exhibe un comportamiento no lineal; en el se pueden efectuar pr.cticas de control de posiciÛn y velocidad del v·stago. El robotmanipulador es un sistema con m' Itiples entradas y salidas que nos permite realizar pr.cticas m-s complejas, ya que es un sistemaaltamenteacopladoyfuertementenolineal. Enelsepueden llevaracabopr-cticas decontrol deposiciÛnyseguimiento de trayectorias.

\section{Uso del sistema de laboratorios a distancia}

Cuando los usuarios acceden al sitio Webdel SLD, lo primero que tienen que hacer es registrarseenel sistemaproporcionandosu nombre de usuario y su contraseÒa. Esto permite tener dos tipos de usuarios: usuarios de $\mathrm{pr}$.cticas yadministradores. Lasfunciones de los administradores $\mathrm{ser} \cdot \mathrm{n}$ comentadas en la
secciÛn 5. Una vez dentro del sistema los usuarios depr-cticaspueden llevara cabovarias operaciones, tales como ver las p-ginas de teorla, contactarcon los desarrolladoresdelsistemaasìcomorealizarlas pr.cticas disponibles en el mismo. Todas estas $p \cdot$ ginas se encuentran localizadas en el servidor Web, el cual es com 'n para todas las pr.cticas. DespuÈs de que elusuario aseleccionadoalgunapr ctica, se le muestra una p.gina Webque contiene el diagrama de bloques de la pr ctica a realizar, una explicaciÛn delasimbologlautilizadaenla misma, enlacesalasp·ginas de teorla asociadas y un formulario en donde el usuario puede modificar los par-metros del controlador, el tiempo de muestreo, elvalor de las referencias ocrearuncontrolador.

\subsection{Prácticas con controlador predefinido}

En este tipo de pr.cticas el usuario sÛlo necesita un navegador de Web (Netscape, Internet Exploreru otro) para acceder al sitio Web del SLD. Una p-gina Web cl-sica para realizar este tipo de pr.ctica es mostrada en la Figura2. Enella aparece un formulario, donde sedebenespecificar los datos delcontrolador, el tiempo de muestreo o cualquier otro dato asociado a la pr-ctica que se desea realizar.

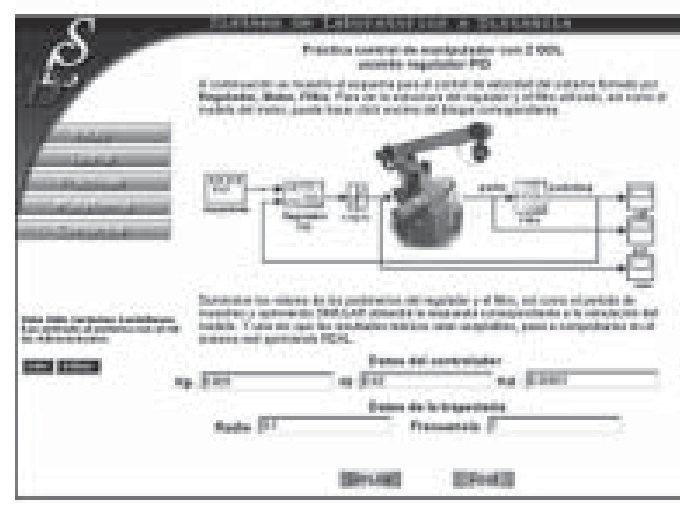

Figura 2: P.gina Web y formulario para realizar $\mathrm{pr} \cdot \mathrm{cticas}$ con controladores predefinidos. 
Adicionalmentese puedeoptarpordosformas derealizaciÛndelaspr-cticas:demanerasimulada, donde se simula la ejecuciÛn y se obtiene una respuestaidealizada de lapr-ctica o de formareal.

\subsection{Prácticas con controlador definido por el usuario}

Cuando el usuario accede a alguna pr.ctica en la que se puede desarrollar el controladora emplear,-es visualizada unap-gina Webcomo la mostrada en la Figura 3.

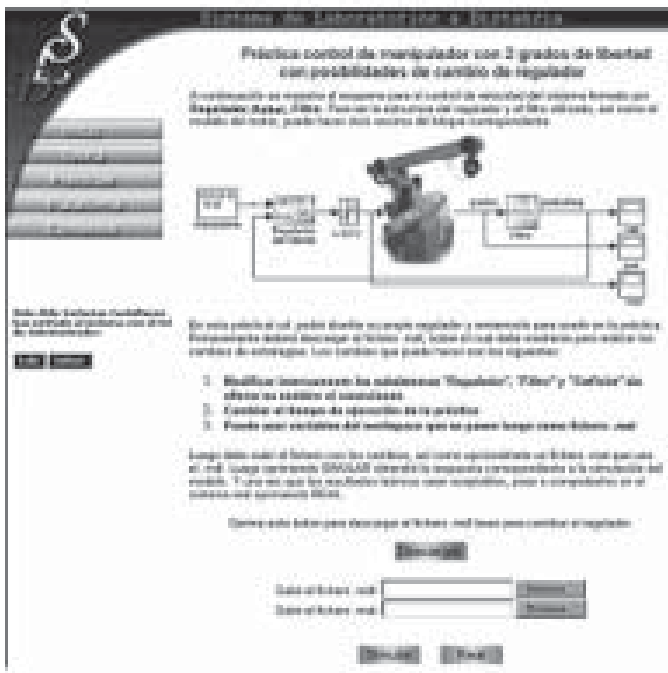

Figura 3: P.gina Web y formulario para realizar pr.cticas con controladores definidos por los usuarios

De ella, el usuario debe descargar un archivo de Simulinkque contiene el diagrama debloques delapr.ctica a realizar. Parallevara cabo este tipo de pr.cticas el usuario necesita tenerinstaladoelsoftware Matlab-Simulinkpara podermodificar el archivo de Simulinkdescargado, tal como se explicara en la secciÛn siguiente.

\section{Creación de controladores}

Una de las caracterìsticas $\mathrm{m} \cdot \mathrm{s}$ importantes del SLD, es que permite a los usuarios lacreaciÛndesuspropios controladores deforma remota. Estos controladores pueden ser creadosutilizandosolo bloques de Simulink, o de manera opcional, una combinaciûn de bloques de Simulink con funciones definidas porelusuario. Paraunmejorentendimientose presentan ejemplos de creaciûn de controladores definidos por el usuario paralos dos casos: primero en un motor de corriente directa y posteriormente para un robot manipulador Asea IRB-6.

\subsection{Creación de controladores utilizando bloques de Simulink}

Cuando el usuario selecciona una pr.ctica con posibilidad de crear un controlador, se le muestra una p·gina Web que contiene un formulario como el mostrado en la Figura 4. En esta p·gina el usuario debe descargarunarchivoquecontieneeldiagrama de bloques de la pr.ctica.

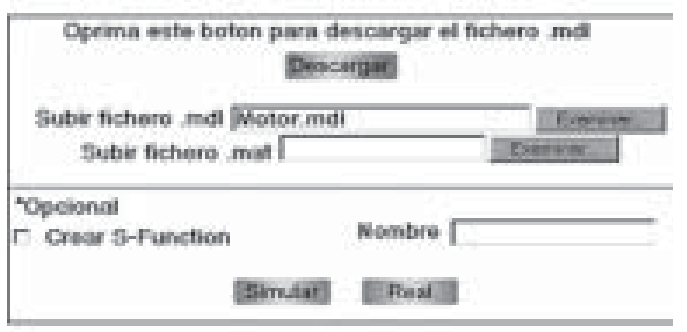

Figura 4: Formulario para realizar pr.cticas con controladores definidos por el usuario utilizando bloques Simulink

En este archivo el usuario debe modificar los subsistemas referencia y controlador utilizandoel software Matlab-Simulink, sinalterar el nombre de las entradas y salidas de los subsistemas tal como se muestra en la figura 
5 , en donde el usuario utiliza una suma de tres seÒalessenosoidalescomoseÒaldereferencia en el subsistema referencia y ha creado un controlador PID dentro del subsistema controlador.

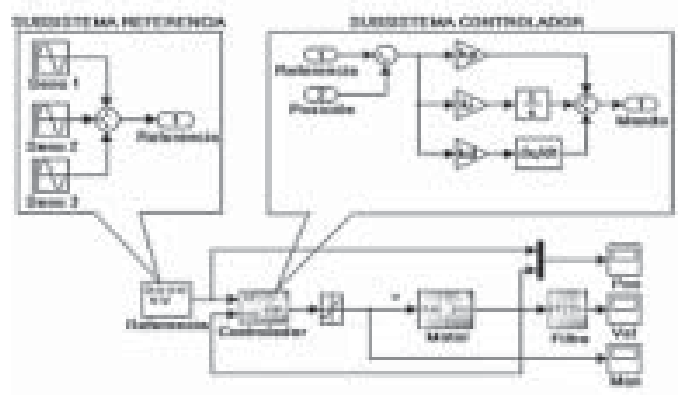

Figura 5: Referencia y controlador PID implementados por el usuario utilizando bloques de Simulink para controlar un motor de corriente directa

Una vez realizada la modificaciÛn, el usuario debe enviar al servidor el fichero de Simulinkmodificadoy decidirsiquiere efectuar una simulaciûn o controlar el proceso real. Cuandosehamandandoacontrolarelproceso real, elSLD realizaprimerounasimulaciÛndela pr.cticaysobrelosdatosobtenidosdelamisma, serealizanunaseriedepruebasparadeterminar sielcontroladorsepuedeimplementarenelsistemareal.Enelcasodelmotordecorrientedirecta estas pruebas se centran en determinar, mediante un an-lisis del espectro de frecuencia, siel sistemapresentaoscilaciones dealtafrecuencia que puedan daÒar al motor mec.nicamente o portemperatura. Unavezdeterminadoesteaspecto el SLD se encarga de implementar el controladorcreado, compilarelsistemautilizandoel Toolbox Real Time Workshop(RTW) yrealizarlapr·cticaentiemporeal.

\subsection{Creación de controladores utilizando bloques de Simulink y S-fuction defi- nida por el usuario}

A travÈs de esta funcionalidad el SLD permite la creaciÛn de controladores complejos, los cuales hacen uso de bloques de Simulinky de S-Functionsescritas en lenguaje C. Cuando el usuario selecciona una pr.ctica con posibilidad decrear uncontroladoryactiva la casilla de creaciÛn de S-Function, se le muestra una $\mathrm{p} \cdot g$ ina $W e b$ que contiene un formulariosimilar al delejemplo anterior (Figura 4) pero que contiene cinco campos adicionales, como el mostrado en la Figura 6. Los campos adicionales son comentados a continuaciÛn:

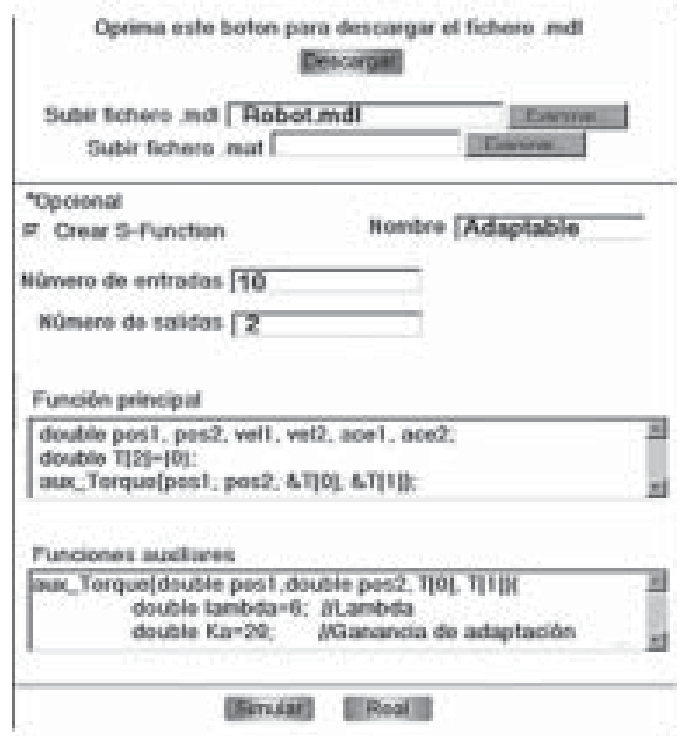

Figura 6: Formulario para realizar pr-cticas con controladores definidos por el usuario utilizando bloques de Simulink y S-Functions

Nombre:Enestecampo se debeespecificar el nombre de la S-Function. Este ser-el nombre del bloque definido por el usuarioenel archivo de Simulink.

Número de entradas: Declara el n' mero de entradas multiplexadas que entraran al bloque definidoporelusuario. 
Número de salidas: Especifica el n mero de salidas demultiplexadas que saldr·n del bloque definido porelusuario.

Función principal: Aquìelusuario debe escribir elcÛdigo dela S-Functionespecificandolasentradasalbloque definido porelusuario como u[0], u[1] ...u[n] y las salidas como y[0], $y[1] \ldots y[n]$. Este cÛdigo debe ser escrito en lenguaje C.

Funciones auxiliares: Enestecampoel usuario puedeescribirladeclaraciÛndelasfunciones auxiliares, las cuales ser.n llamadas desde la funciÛn principal. El cÛdigo debe ser escrito en lenguaje $\mathrm{C}$.

Al igual que en el ejemplo anterior, en estap.gina Webelusuario debe descargarun archivo de Simulinkque contiene el diagrama de bloques de la pr.ctica (robot.mdl en este caso). En este archivo el usuario debe modificar el subsistema controlador utilizando el software Matlab-Simulink, sinalterarel nombre de las entradas y salidas del subsistema, tal comosemuestraenlafigura7, dondeelusuario haimplementado un controlador PD concompensaciÛn adaptable. Este controlador utiliza

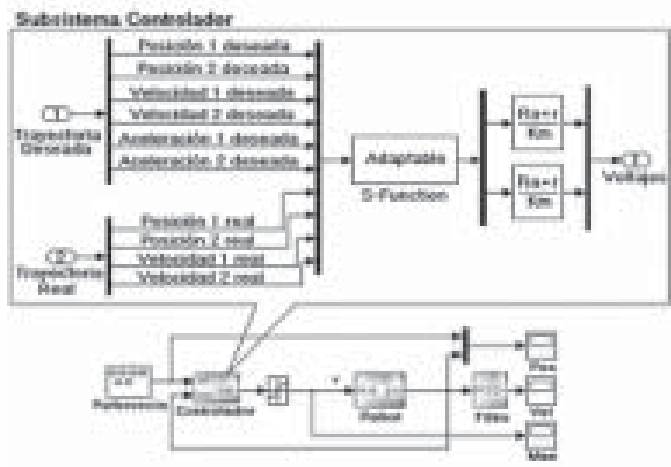

Figura 7: Controlador PD con compensaciÛn adaptable implementado por el usuario utilizando bloques de Simulink y $S$-Functions para controlar el robot manipulador Asea IRB-6 una funciÛn definida por el usuario a travÈs de una S-Function llamada ìAdaptableî en el subsistema controlador. En los cinco campos adicionales del formulario, el usuario deberponerel nombre dela S-Function(enestecaso Adaptable), el n' mero de entradas (10 entradas), eln mero de salidas (2 salidas) yescribir el cÛdigo principal de la funciÛn, asì como las funciones auxiliares que utilizara tal como se muestra en la Figura 6. Una vez realizadala modificaciÛnydefinidala S-Function, elusuario debe decidirsiquiere realizarunasimulaciûno controlar el proceso real. Cuando se ha mandando hacontrolar el proceso real, elSLD realiza primero una simulaciûn de la pr.ctica asì como una serie de pruebas, que en el caso del robotmanipulador, est·ncentradas en tres aspectos:

1. Verificarquelatrayectoriacartesiana no sobrepase el espacio de trabajo del robot manipulador.

2. Verificar las posiciones articulares, de manera que no sobrepasen los IÌmites mec-nicosdecadaarticulaciÛn.

3. Comprobar que el sistema en lazo cerrado, no presente oscilaciones de alta frecuencia que puedan desajustar mec-nicamente al robot y/o daÒar los actuadores del mismo.

Unavez determinados estos aspectos, el SLD se encarga de implementar el controlador, compilar el sistema utilizandoel Toolbox RTW y realizar la pr.ctica en tiempo real. En caso de que el SLD detecte que el controlador desarrollado representa un riesgo parael robot manipulador, seleinformaalusuariolacausay le son proporcionados los datos y graficas de la simulaciÛnrealizadaparaquepuedaexaminar elcomportamientodelsistema. 


\section{$5 \quad$ Administración del SLD}

Para implementar la administraciÛn de horarios y usuarios del SLD, fue necesaria la creaciÛn y gestiÛn de las bases de datos del servidor del sistema, asì como la programaciÛn de la interfaz de usuario en ASP. SeutilizÛSQL Server 2000 como mecanismo gestor de la base de datos, ya que es un sistema muy completo formado por un conjunto de componentes desarrollados para el almacenamiento y an.lisis de grandes sitios Web.

\subsection{Base de datos del sistema}

La base de datos deISLD est · formada por las siguientes tablas:

User: Almacena los datos de todos los usuariosque han accedidoalSLD, incluyendo el nombre de usuario y la contraseÒa.

Practices: Guarda todas las pr.cticas disponibles en el sistema, su nombre, la ubicaciÛn desup·gina Webasociada, etc.

Group: Engloba todos los grupos de estudiantes que existen en el sistema, para poder administrar, en caso de ser necesario, los tiempos de los laboratorios asignados a cadagrupo.

Schedule: Contiene todos los horarios reservados para uso docente, asì como las $\mathrm{pr} \cdot \mathrm{cticas}$ que han sido elegidas en los diferenteshorarios.

Estassonlastablasfundamentalesque utiliza el SLD, adem.s de otras tablas que se utilizan para las relaciones entre ellas Por ejemplo UserByGroupy UserRole ByPractices talcomosemuestraen lafigura 8. Losusuarios delSLD pueden tener rol de administrador del sistema o de usuario. Mientras que por cada pr-ctica pueden tener rol de registrado, estudiante, profesoroadministrador.

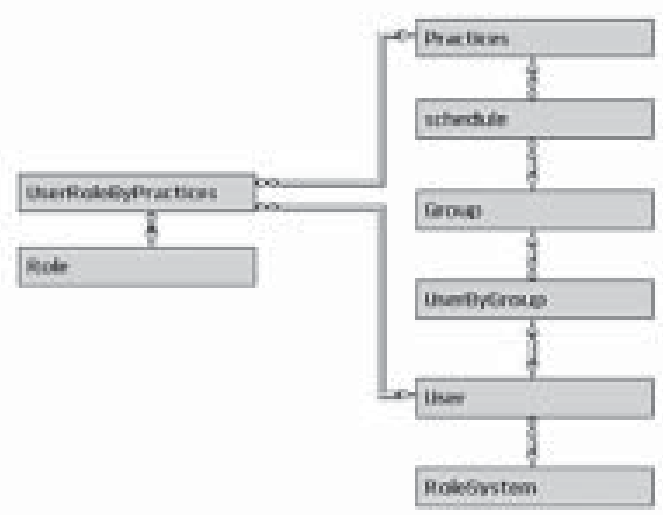

Figura 8: RelaciÛn entre las distintas tablas que conforman la base de datos del SLD

\subsection{Interfaz de usuario y de administración}

Las p.ginas Web din-micas fueron creadas utilizando la tecnologl̀a ASP. Esto incluye alas $p \cdot g i n a s$ de acceso a las pr.cticas, teorla y administraciÛn. Solo los usuarios con permisos deadministraciÛn podr.nverlap·ginadeadministraciÛn, pueselbotÛn deacceso aparecer-solo para los que tengan ese rol en elsistema. El administradormediante estap-ginapuedegestionarlosusuarios, laspr-cticas, lasp·ginas deteorla, losgrupos delaboratorios para uso docente y asignar, en caso de ser necesario, el horario de acceso alas pr.cticas. Losusuarios, unavezqueaccedenal sitio Web, pueden registrarse en el sistema si no lo han hecho, mediante unap ginaWebquecontiene todoslosformulariosnecesarios. Sisecomete alg ' $n$ error en el registro de usuarios el sistema informara al usuario, como por ejemplo, si el nombre de usuario ya fue elegido, si la direcciÛn de correo no es valida o si la contraseÒa no cumple con los requisitos de tamaÒo (8 caracteres como mìnimo). Es indispensable para usar el sistema estar re- 
gistrado e introducir el nombre de usuario y la contraseÒa. Una vez autenticado, el usuario puede acceder a las p-ginas de teorla y a las pr.cticas que estÈn disponibles en ese momento. En caso de existir una pr.ctica con horario reservado para uso docente y el usuario no pertenecer al grupo de laboratorio correspondiente, lapr-cticaaparecer-deshabilitada y se le informar que est - siendo usada por un determinado grupo y que deber-esperaraque el grupo termine de utilizarla para poder realizarlapr·ctica.

El numero de usuarios que pueden acceder simult-neamente al SLD no esta limitado, sin embargo, el numero de peticiones de pr.cticas que consigue atender simult. neamente el SLD si esta limitado y es configurable por los administradores del sistema. Actualmente esta configurado para atender hasta veinte peticiones de pr.cticas, las cualessepueden llevaracaboen distintasestaciones de trabajo. Cuando existe un $n$ ' mero mayor de peticiones que el configurado en el sistema, elSLDinformaal usuario que elservidor esta congestionado y que deber esperar un tiempopara poder realizarla pr.ctica.

\section{Experiencias de uso del SLD}

Durante el aÒo 2003 se realizaron las primeras pruebas del SLD desde MÈxico y EspaÒa,conelfindeimplementarcontroladores en los dispositivos localizadosen Cuba. EISLD se utilizo para que los alumnos realizaran pr-cticas, de forma remota, en cursos de postgrado en las materias de teorla avanzada de controlyrobÛticaenel Instituto TecnolÛgicode Minatitl'nen MĖxico. Duranteestas pr.cticasel sistematrabajÛdeformasatisfactoria, conaproximadamente 30 usuarios utilizando el sistema, pero presentaba tiempos de esperagrandes entre el momento de mandar a realizar la pr-ctica y el momento de recibir la respuesta, por lo que se empezÛ a trabajar en dos vìas:

1. Modificar el formato de las im.genes para tener una carga $\mathrm{m} \cdot \mathrm{s} \mathrm{r} \cdot$ pida en las $\mathrm{p} \cdot \mathrm{gi}$ nas del sitio Web, asì como revisar los algoritmos encargados de implementar los controladores, de forma que hubiera una reducciÛn en el tiempo de procesamiento.

2. Realimentar al usuario los datos del proceso controlado, para que durante la espera de la p gina de respuesta, pueda visualizar en un applet Java el estado del proceso durante el control. Esto permitir queelusuario sepa que sÈ esta procesandola informaciÛny no piense que el sistema esta ìcolgadoî.

Lasmodificaciones referentesal primer punto se encuentran ya terminadas, mientras que las del segundo punto se encuentran actualmente en fase de desarrollo.

A partir del presente aÒo elSLD ha sido utilizado para realizar pr cticas en cursos de identificaciÛnycontroldeaccionamientosenlas carreras de Ingenierla Mec.nicae Ingenierlaen Autom tica en la Universidad Central ìarta Abreuî de las Villas y en la Universidad de Cienfuegos, ambas localizadas en Cuba. Durante estas lecciones los usuarios mostraron ungran interÈsporcontrolardispositivos deformaremotaatravĖs de Internet. Trasunabreve explicaciÛnsobrelosfundamentosteÛricosde la pr.ctica, los usuarios se registraron en el mismo y comenzaron a realizar experimentos en menos de cinco minutos. Uno de los aspectos que $\mathrm{m}$. $\mathrm{s}$ estimulo a los estudiantes fue poder evaluar las diferencias entre los modelos matem ticos y los dispositivos reales. En promedio con quince alumnos por grupo, se realizan entre 60 y $80 \mathrm{pr} \cdot$ cticas en menos de 30 minutos, lo que da una idea de la alta tasa de 
aprovechamientode losequiposal accederlos de forma remotautilizando Internet.

\section{Conclusiones}

EI SLD proporciona a los usuarios la facilidad de uso de paquetes como Matlaby SimulinkenconjuntoconelToolboxRTW para la creaciÛn y prueba de controladores de forma remota en dispositivos reales. Mediante estemecanismoes posiblelaimplementaciûn de controladores que utilicen algoritmos complejos, los cuales pueden ser creados de una forma sencilla utilizando la potencia y flexibilidadque proveen las S-Functionsescritas en lenguaje $\mathrm{C}$.

Comoel software deISLDesf·cilmente adaptableanuevosprocesos, laincorporaciÛn de nuevos dispositivos para efectuar experimentosse puederealizarf $\cdot$ cilmente. Debidoa que el sistema esta aun en fase de desarrollo, el numero de dispositivos disponibles es limitado (un motor de corriente directa, un robot manipulador y un cilindro electro neum·tico), pero se esta trabajando para en un futuro, en colaboraciÛnconotrasuniversidades, incorporarnuevos procesos que incluyan experimentos paraelcontrol de temperatura, el controlde nivel, entreotros experimentos. Deestaforma, variasuniversidadesycentros deinvestigaciûn pueden compartir recursos materiales costosos y los usuarios pueden, desde cualquier parte del mundo, realizar pr.cticas de control con dispositivos que est $\cdot n$ geogr $\cdot$ ficamente distantes.

EI SLD cuenta con un sistema de administraciÛn, tanto de pr.cticas como de usuarios, elcualutiliza SQL Server 2000 como gestor de base de datos ya que es un sistema fiable, eficiente y seguro. Este sistema de administraciÛn permite incorporar, editaroeliminar tanto pr.cticas como a usuarios del sistema, asìcomoposibilitar laasignaciÛn de distintas prioridades a los grupos o usuarios en determinados horarios. De esta forma, si un profesor requierequesusalumnosutilicenuna determinada pr.ctica a una cierta hora, los administradoresdelsitio pueden reservaresahora exclusivamente para esegrupo ousuario, y todos los dem.s usuarios estar.n imposibilitados de usar esa pr.ctica en ese horario reservado.

La tecnologla ASP fue utilizada para realizar toda la programaciÛn de las $p \cdot g i n a s$ Web din·micas, logr·ndose con esto incrementar la seguridad del SLD ante eventuales accesos de usuarios no registrados o eliminados por los administradores.

Asimismo la incorporaciÛn en un futuro deunamayorymejorrealimentaciÛnalusuarioa travĖs de latecnologla Java, har.al sistemam.s interactivoyestimulanteparalosusuarios.

Referencias

ALHALABI, B., ANANDAPURAM, S., HAMZA, K. Real laboratories: An innovative repartee for distance learning!. En: JOURNAL OF OPEN PRAXIS, v.2, p.24-30, 2000.

ALHALABI, B., HAMZA, M. K., HSU, S., ROMANCE, N. Virtual labs vs. remote labs: Between myth \& reality. En: 7TH FLORIDA HIGHER EDUCATION CONSORTIUM, Deerfield Beach, Florida, 1998.

AUSSERHOFER, A., Web-based teaching and learning: a panacea?. En: IEEE MAGAZINE, p.92-96, 1999.

BAUCHSPIESS, A., GUIMARAES, B., GOSMANN, H. L. Remote experimentation on a three coupled water reservoirs. En: IEEE INTERNATIONAL SYMPOSIUM ON INDUSTRIAL ELECTRONIC, Rio de Janeiro, Brasil, 2003. 
CASINI, M., PRATTICHIZZO, D., VICINO, A. E-learning by remote laboratories: A new tool for control education. En: 6TH IFAC SYMPOSIUM ON ADVANCES IN CONTROL EDUCATION, Oulu, Finlandia, 2003.

CHANG, T., HUNG, D. Web based distance experiments: Design and implementation. En: INTERNATIONAL CONFERENCE ON ENGINEERING EDUCATION, Taipei, Taiwan, 2000.

CHAUDHURI, A., BHATTACHARYYA, C., AKULI, A. Internet based instrumentation with multimedia features: some developments for technical education. En: INTERNATIONAL CONFERENCE ON MULTIMEDIA TECHNOLOGY, Filipinas, 2003.

CHICULITA, C., FRANGU, L. A Web based remote control laboratory. En: THE 6TH WORD MULTICONFERENCE ON SYSTEMICS, CYBERNETICS AND INFORMATICS, Orlando Florida, 2002.

DIXON, W. E., DAWSON, D. M., COSTIC, B. T. Towards the standardization of a Matlab-based control system laboratory experience for undergraduate students. En: AMERICAN CONTROL CONFERENCE, Arlington VA, 2001.

DOMINGUEZ, M., MARCOS, D., REGUERA, P., GONZALEZ, J. J., BL;ZQUEZ, L. F. Connection pilot ¥plant to the Internet. En: PROCEEDINGS OF IFAC INTERNET BASED CONTROL EDUCATION, Madrid, EspaÒa, 2001.

FRANGU, L., CHICULITA, C. Remote laboratory allowing full-range student designed control algorithm. En: 9TH IEEE INTERNATIONAL CONFERENCE ON ELECTRONICS, CIRCUITS AND SYSTEMS, Dubrovnik, Croacia, 2002.

GARCIA, R. M., WORNIE, F., STEWART, B. G., HARRISON, D. K. Real-time remote network control of an inverted pendulum using ST-RTL. En:32ND ASEE/IEEE FRONTIERS IN EDUCATION CONFERENCE, Boston, MA, 2003.

HAHN, H. H., SPONG, M. W. Remote laboratories for control education. En: PROCEEDINGS OF THE 39TH IEEE CONFERENCE ON DECISION AND CONTROL, Sydney Australia, 2000.

JUNGE, T. F., SCHMID, C. Web-based remote experimentation using a laboratory-scale optical tracker. En: PROCEEDINGS OF THE AMERICAN CONTROL CONFERENCE, Chicago, Illinois, 2000

KO, C. C., CHEN, B. M., CHEN, J., ZHUANG, Y., TAN, K. C. Development of a Web-based laboratory for control experiments on a coupled tank apparatus. En: IEEE TRANSACTIONS ON EDUCATION, pp.76-86, 2001

POINDEXTER, S. E., HECK, B. S., Using the web in your courses: What can you do? What should you do?. En: IEEE CONTROL SYSTEM MAGAZINE, p.83-92,1999.

SCHMID, C. The virtual control VCLab for education on the web. En: PROCEEDINGS OF THE AMERICAN CONTROL CONFERENCE, Philadelphia, Pennsylvania, 1998.

SEBASTIAN, D. Control learning: Present and future. En: 15TH IFAC WORLD CONGRESS, Barcelona, EspaÒa, 2002.

SHOR, M. H. Remote-access engineering educational laboratories: Who, what, when, where, why, and how ?. En: PROCEEDINGS OF THE AMERICAN CONTROL CONFERENCE, Chicago, Illinois, 2000.

TREVELYAN, J. Towards cost effective on-line laboratories. En: WORLD CONGRESS NETWORKED LEARNING IN A GLOBAL ENVIRONMENT, Berlin, Alemania, 2002

Recebido em 30/03/2004

AceitoparapublicaÁ,,oem20/07/2004

MSc. Aldo R. Sartorius Castellanos

Dr. Luis Hern·ndez Santana

Dr. Rafael Aracil Santonja

MSc. Ernesto Rubio Rodrìguez

MSc. Iv·n Santana Ching

UniversidadCentralìMartaAbreuîdeLas Villas

DepartamentodeAutom·ticaySistemasComputacionales

CarreteraaCamajuani Km.5 $\Omega$ SantaClara, VillaClara, Cuba

E-mail:sartorius@uclv.edu.cu 